\title{
ON THE RELATION BETWEEN MOVING MAGNETIC \\ FEATURES AND THE DECAY RATES OF SUNSPOTS
}

\author{
CORNELIS ZWAAN \\ Astronomical Institute, Utrecht, The Netherlands
}

\begin{abstract}
Bumba (1963) inferred from plots of areas of sunspot groups against time that stable spots may show a phase of slowest decay which is independent of the spot's area. From the Greenwich Photoheliographic Results we analysed the areas of individual spots. Indeed there is a clear lower limit in the decay rates close to Bumba's value $\mathrm{d} A / \mathrm{d} t \simeq-4.0 \times 10^{-6}$ visible hemisphere/day $\hat{=}-5.0 \times 10^{15} \mathrm{~cm}^{2} \mathrm{~h}^{-1}$. However, only a small fraction of regular spots and an even smaller fraction of the irregular spots pass through the phase of slowest decay.
\end{abstract}

A slow erosion by some supergranular velocity field cannot explain why the rate is independent of area. Rates independent of area are compatible with a model in which the decay is ultimately determined by diffusion across a relatively thin cylindrical current sheet, provided that the ratio between the thickness $\Delta R$ of the current sheet and the total radius $R$ of the flux tube remains constant during the decay (Gokhale and Zwaan, 1972). Such a radial similarity $\Delta R / R=$ constant fits the radial similarity in the observable sunspot structure. If the diffusion occurs by purely Ohmic dissipation, then the current sheet should be extremely thin: $\Delta R / R$ between $10^{-5}$ and $10^{-4}$.

From a plausible magnetic field configuration $\mathbf{B}(r)$ and the rate of slowest area decay follows a flux decay rate $\mathrm{d} \Phi / \mathrm{d} t \simeq-6.5 \times 10^{18} \mathrm{Mx} \mathrm{h}{ }^{-1}$. This value comes very close to the value $\mathrm{d} \Phi / \mathrm{d} t \simeq-1.0 \times 10^{19} \mathrm{Mx} \mathrm{h}^{-1}$ derived by Harvey and Harvey (1973) from magnetic features moving away from two sunspots. If indeed these two spots were in the mode of slowest decay, then it would follow that the diluted magnetic flux crossing the current sheet is carried away in discrete and detectable flux tubes. Certainly rapidly decaying spots often show a fragmentation into magnetic features larger than the socalled moving magnetic features. However, there is no clear picture yet as to what photospheric and chromospheric phenomena around sunspots accompany the decay processes at various rates.

There are some indications that the decay rates of sunspots are related with the structure in the umbrae (Zwaan, 1968). Spots with umbrae showing dark cores without clear structure decay slowly. Umbrae with a dense pattern of bright structures, umbral dots or light bridges or both, seem to indicate rapidly decaying spots. Implications are briefly discussed.

Simultaneous observation of the three aspects of decay, viz.

(i) the photospheric and chromospheric phenomena around the spot (e.g., fragmentation, moving magnetic features);

(ii) the decay rate of sunspot area $\mathrm{d} A / \mathrm{d} t$; 
(iii) the fine structure in the sunspot, would substantially contribute to a better understanding of sunspot structure down in the convection zone and of the mechanisms distributing the magnetic flux over the photospheric surface. When seeing permits the observation of the first aspect then the second and third aspects may be secured without great difficulty.

An account of the investigation of decay rates of sunspots is planned as a paper in the series 'The Structure of Sunspots', to be published in Solar Physics.

\section{References}

Bumba, V.: 1963, Bull. Astron. Inst. Czech. 19, 91.

Gokhale, M. H. and Zwaan, C.: 1972, Solar Phys. 26, 52.

Harvey, K. and Harvey, J.: 1973, Solar Phys. 28, 61.

Zwaan, C.: 1968, Ann. Rev. Astron. Astrophys. 6, 135.

\section{DISCUSSION}

Smith: In your decay phase model you have suggested that you have a very thin current sheet. I imagine that the field of the sunspot is vertical, so I don't see how the current sheets are formed. What kind of field do we have around the sunspot?

Zwaan: Very thin current sheets follow from the assumption of purely ohmic dissipation. If you add extra resistivity, for example, because of turbulence the thickness of the current sheet will grow in proportion. I don't have an idea how the current sheet comes about but it does fit in with the radial similarity in observed sunspot structure and with the lower limit on the sunspot decay rates. A second indication is that the umbra is a rather uniform thing, apart from fine structure that may or may not be present. This suggests that the whole column below the umbra is rather uniform within that column. May I comment on one more thing. I think it is not difficult to observe the decay rates of sunspots accurately from a continuum signal from the magnetograph. The slow decay rate corresponds to one square arcsecond per hour.

Giovanelli: If I understood you correctly, your final diagram showed the umbra of a sunspot in which one portion was dark and another portion contained a lot of little dots, which I assume to be umbral granulation. Your suggestionis that the umbral granulation stays there in more or less the same manner for quite some time. However the lifetime of umbral granules is only of the order of one hour. Isn't there some discrepency here.

Zwaan: I did not say that exactly the same umbral dots are staying there all the time but that a certain area of the umbra shows visible fine structure where the other parts remain dark without observable structure. I think that the irregularity in the appearance of the umbral fine structure indicates that umbral dots are not some convective mode necessary for the physical health of the sunspot.

Bumba: Sunspots that are very regular often dissipate in such a way that granules develop inside the umbra along its border. There are many cases when pieces of umbra or penumbra travel from the sunspot through the intergranular space. This is a very interesting phenomena. I have tried to obtain a movie of it but I have not yet succeeded. My impression is that you have a dark piece of matter pushed out of the sunspot through intergranular space. This dark matter eventually disappears in the intergranular field. 